\title{
Polymer structure effect on gas permeation characteristics in polyarylates
}

\author{
S S KULKARNI \\ National Chemical Laboratory, Pune 411008 , India

\begin{abstract}
This paper reviews recent investigations of the factors which control gas transport in a series of highly aromatic polyarylates. The polymer physical properties which correlate with its intrinsic separation characteristics were elucidated by studying well-defined families of polyarylates. Molecular modelling was used to understand the effects of various substitutions in the polymer structure and the mechanism through which the polymer properties are affected. The effect of polymer chain mobility, conformation and packing density on the permeation characteristics are discussed.
\end{abstract}

Keywords. Permeation; polyarylates; gas separation.

\section{Intrcduction}

Polymers which are used to fabricate gas separation membranes need to meet several criteria such as high permeability to the desired gas, high selectivity, ability to be formed into useful membrane configurations, and resistance to processing conditions of high pressure, moisture, etc. The most important criteria are the polymer permeation characteristics. The economic viability of membrane gas separation depends greatly on membrane productivity, i.e. flux per unit membrane surface area. This is a function of both the intrinsic permeability of the membrane material and the ability to form it into a thin, defect-free film. The permselectivity of the membrane material establishes the maximum separation achievable.

The ideal membrane material would combine high permeability with high permselectivity. Unfortunately, polymers in general show an inverse correlation between these two properties. Polymers with high permeabilities tend to have low selectivities, and vice versa. Identification of new materials which overcome this natural trend is required for improving the cost effectiveness of membrane gas separations.

Recently, systematic studies of polymer structure-permeability relationships in several families of glassy polymers have led to the identification of new materials for gas-selective membranes which combine high permeability and permselectivity. Such studies have been carried out for polyimides (Kim et al 1988; Stern et al 1989; Coleman and Koros 1990), polycarbonates (Anand et al 1989; Schmidhauser and Longley 1990), polysulphones (McHattie et al 1991; Houde et al 1994) and polyarylates (Sheu and Chern 1989; Charati et al 1991a). Polyarylates in particular are a promising class of membrane materials particularly for air separation. The potential of using polyarylates as a membrane material can be seen in the study (Barbari et al 1989) of four types of polymers based on bisphenol-A (Bis-A), viz. polyarylate, polycarbonate, polysulphone and polyetherimide. The gas permeability of the polyarylate based on bisphenol $\mathrm{A}$ and equimolar mixtures of isophthalic and terephthalic acids was higher than in the other polymers. 


\subsection{Polymer characteristics which influence transport properties}

It would be desirable to understand the effect of various polymer characteristics on permeability. Transport properties of gases are known to depend on structural parameters like packing density in the polymer, polar group density, chain rigidity, and molecular mobility (as determined by glass, $T_{\mathrm{g}}$, or lower transition, $T_{y}$, temperatures). Establishing polymer structure-permeability correlations is important for rational design of new polymers for specific gas-separation applications. This paper reviews some of our recent work with polyarylates towards this goal.

Recent work has identified several polymer characteristics which as a general rule are found to affect either the permeability or the permselectivity. The effect of decreasing packing density of the polymer matrix on increasing diffusivity and permeability has been shown in several types of polymers (Kim et al 1988; Stern et al 1989; Charati et al 1991a). It has also been shown that simultaneous inhibition of the interchain packing and restriction of the intrasegmental mobility as a result of substitution by a bulky group results in a polymer structure which leads to high permeability and selectivity (Hoehn and Richter 1980; Kim et al 1988). The opposing effects of polymer polarity (increased interchain packing coupled with increased gaspolymer interaction) on gas permeability have been reported (Muruganandam et al 1987). Other parameters investigated include the dielectric constant (Matsumoto et al 1993) and charge transfer complex formation (Stern et al 1989).

\subsection{Rational design of polymer structure}

While definitive a priori design of a polymer with specified permeation characteristics is not yet possible, much progress has been made towards this goal. As mentioned above, there are now consistent data correlating various polymer characteristics with permeation properties. However, it is still difficult to relate these properties to the chemical repeat-unit structure of the polymer. Empirical correlations exist where group additivity methods have been used for correlating polymer permeabilities. Such correlations (e.g. the well-known 'permachor' method proposed by Salame 1967) are useful for describing variation over several magnitudes of permeability values; however, these do not give the fine insight required for deciding how to modify a given polymer. Semi-empirical models exist which relate polymer characteristics to transport phenomena. Such models based either on activation-energy concepts (Pace and Datyner 1979) or on free-volume models (Kulkarni and Stern 1983) can be used to predict diffusivities if some polymer physical characteristics are known. However, these models do not have a direct relationship with polymer structure.

\section{Present approach}

To increase our understanding of the effect of polymer structure on permeation properties, the four-step approach followed by us is given below:

A. Systematic synthesis. Families of polyarylates with systematic variations in the chemical structure have been synthesized. In the case of polyarylates which are prepared by the condensation reaction of aromatic diols and acids, it is possible to 
modify either reactant. The modified polymers can then be compared with the base material. Starting with the polyarylate formed from bis-A and equimolar mixtures of terephthalic and isophthalic acids as the base material, two families of new polyarylates synthesized with systematic modifications of the bisphenol are shown in figures 1 and 2 (Charati et al 1991a). In figure 1, one methyl group at the bridge carbon atom is changed to either a longer flexible alkyl group (ethyl), a branched alkyl group (isobutyl), an aromatic group (phenyl), or a polar alkyl group (methyl propionate). In figure 2 , the bisphenol phenyl rings are substituted with either nonpolar (muthyl) or polar $(-\mathrm{Br})$ groups. This substitution pattern may be either symmetric (tetramethyl) or asymmetric (dimethyl).<smiles>[R]C(C)(c1ccc(OC)cc1)c1ccc(OC(=O)c2ccccc2)cc1</smiles>
i) $\mathrm{R}=-\mathrm{CH}_{3}(\mathrm{PBis}-\mathrm{A})$
ii) $\mathrm{R}=-\mathrm{CH}_{2}-\mathrm{CH}_{3}(\mathrm{PBis}-\mathrm{MEK})$
iii) $\mathrm{R}=-\mathrm{CH}_{2}-\mathrm{CH}_{\mathrm{CH}_{3}}^{-\mathrm{CH}_{3}}(\mathrm{PBis}-\mathrm{MIBK})$
iv) $R=-\bigcirc(P B i s-A c e t o)$
v) $\mathrm{R}=-\mathrm{CH}_{2}-\mathrm{CH}_{2}-\stackrel{\mathrm{C}}{\mathrm{C}}-\mathrm{O}-\mathrm{CH}_{3}$ (PBis Lev)

Figure 1. Structure of bisphenol bridge carbon mono-substituted polyarylates.<smiles>[R]c1cc(C(C)(C)c2cc([R])c(OC(=O)c3ccccc3)c([R])c2)cc([R])c1OC</smiles>
i) $\mathrm{R}=\mathrm{R}^{\prime}=-\mathrm{CH}_{3}(\mathrm{PTMBis}-\mathrm{A})$
ii) $\mathrm{R}=-\mathrm{H}, \mathrm{R}^{1}=-\mathrm{CH}_{3}(\mathrm{PDMBis}-\mathrm{A})$
iii) $\mathrm{R}=\mathrm{R}^{1}=-\mathrm{Br}(\mathrm{PTBr} \mathrm{Bis}-\mathrm{A})$

Figure 2. Structure of bisphenol phenyl ring substituted polyarylates. 
B. Polymer characterization. The synthesized polymers are characterized for various physical properties of interest and these are compared with the permeation properties.

For example, in the first series described above (figure 1), consisting of bridgesubstituted polyarylates, the interchain spacing ( $d$-spacing or $d_{\text {sp }}$ measured by WAXD) is kept constant. The $>\mathrm{C}=\mathrm{O}$ group density and the solubility parameter are also relatively unchanged. In this series the effects of different types of substitutions on the glass transition temperature, $T_{\mathrm{g}}$, and the $\gamma$ transition temperature and correspondingly on permeability can be seen. In this manner, the effect of chain rigidity can be examined in isolation from other variables.

In the second series, of ring-substituted polyarylates, the effects of symmetry and polarity of the substituent groups is investigated. These substituents cause varying amounts of changes in all four parameters, viz. the $d$-spacing, the $>\mathrm{C}=\mathrm{O}$ group density, and the glass and $\gamma$ transition temperatures.

C. Heuristic rules. Some generalizations can be made relating the physicalproperty variations with the permeation-property changes in the polymer series. Such rules may be useful if applicable over a wide range of polymers. In the case of polyarylates, it is possible to describe the effects of polymer chain mobility, polarity and packing density on the permeation characteristics.

D. Molecular modelling. Molecular modelling can be used to explore the mechanism by which chemical structure affects physical properties in order to gain a more meaningful insight and also to guide future synthesis. Because of their highly glassy, amorphous nature, polyarylates are not easy to characterize. In particular, molecularlevel information is difficult to obtain. In such cases, molecular modelling can be used to understand the effects of various substitutions in the polymer structure and the mechanism through which polymer properties are affected.

\section{Heuristic rules relating polymer transport properties with other characteristics}

Some general rules are discussed below and illustrated with examples from the work with polyarylates.

\subsection{Effect of packing density}

Modifications in polymer structure which reduce the packing density lead to a decrease in selectivity and increase in permeability. This result is also predicted by both activation-energy and free-volume models of gas transport. The packing density of the polymer can be characterized in two common ways: (i) by wide-angle $\mathrm{X}$-ray diffraction (WAXD), (ii) by density measurements. The WAXD spectra of amorphous polyarylates show a broad peak; the peak maximum is used to calculate an average intersegmental distance, $d_{\mathrm{sp}}$, characteristic of that polymer. Density measurements in combination with group additivity methods to calculate the van der Waals volume of the polymer segment can be used to calculate the free volume of the polymer.

Both the free volume and the $d_{\mathrm{sp}}$ are measures of the packing density of the polymer; however Charati et al (1991a) have indicated that for several types of highly glassy 


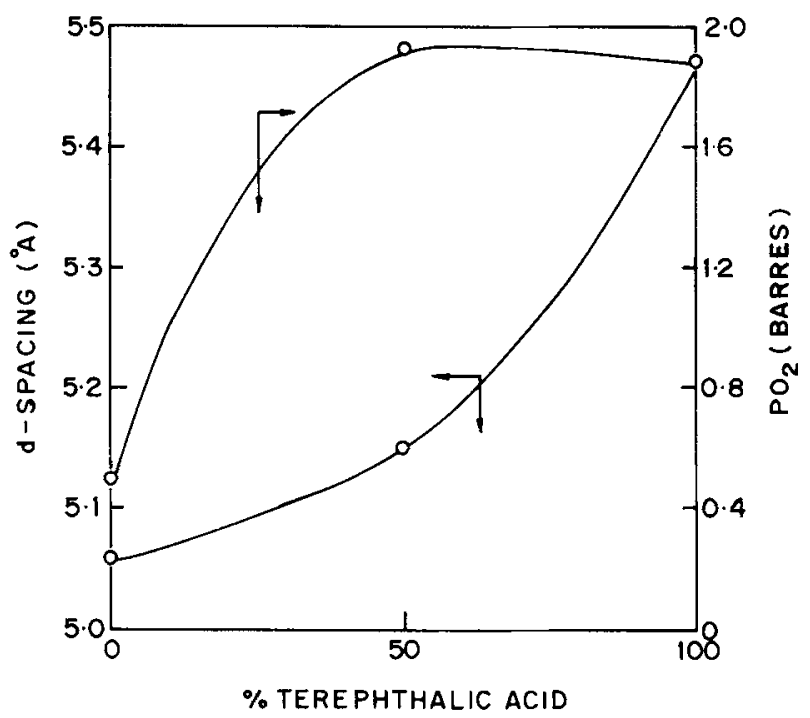

Figure 3. $\mathrm{O}_{2}$ permeability and $d$-spacing as a function of terephthalic acid content in polyarylates based on bisphenol-A and iso/terephthalic acids.

polymers, the $d_{\mathrm{sp}}$ correlates better with the permeability than the free volume. The difference may arise because of errors in calculating the van der Waals volume by the group additivity method.

An example of a correlation between $d_{\text {sp }}$ and permeability is shown in figure 3 . Most polyarylates made with isophthalic and terephthalic acids show increasing $d_{\mathrm{sp}}$ values as the proportion of terephthalic acid is increased; there is a corresponding increase in permeability. Similar correlations can be found for polyimides (Coleman and Koros 1990).

A general trend is that permselectivity decreases with increasing $d_{\mathrm{sp}}$; this is to be expected as a more open polymer matrix will have less ability to discriminate between penetrants on a steric basis. A more subtle effect may also be useful: the increase in permeability of various gases appears not to be uniform with increasing $d_{\text {sp }}$ values. In some ranges of $d_{\mathrm{sp}}$ values, the free-volume distribution may change so as to allow larger increase in permeability of smaller penetrants, e.g. He, while the permeability of larger molecules, e.g. $\mathrm{N}_{2}$, may increase by a smaller amount, thus resulting in a selectivity increase for smaller molecules. This result is the reverse of the predictions from either activation-energy or free-volume models in which the increase in permeability with decreasing packing density would be more for larger molecules. However, neither of these theories account for the concept of free-volume distribution.

The $d_{\mathrm{sp}}$ can also be used to characterize the increase in permeability on plasticization of the polymer from exposure to high-pressure gases with high solubility, e.g. $\mathrm{CO}_{2}$. The change in permeability profiles with increasing pressure can be related to the increase in $d_{\mathrm{sp}}$ for several classes of polymers (Houde et al 1992).

\subsection{Effect of chain rigidity}

The chain rigidity is characterized by the glass transition temperature $T_{\mathrm{g}}$ or lower transition temperature $T_{y}$, measured using dynamic mechanical analysis (DMA) or 
Table 1. Physical properties and $\mathrm{O}_{2}$ permeability of bridge-substituted polyarylates.

\begin{tabular}{|c|c|c|c|c|c|}
\hline \multirow[b]{2}{*}{ Bisphenol } & \multirow[b]{2}{*}{$\begin{array}{c}d \text {-spacing } \\
(\AA)\end{array}$} & \multirow{2}{*}{$\begin{array}{l}\text { Solubility } \\
\text { parameter } \\
(\delta)\end{array}$} & \multicolumn{2}{|c|}{$\begin{array}{l}\text { Transition } \\
\text { temperatures }\end{array}$} & \multirow{2}{*}{$\begin{array}{c}\text { Permeability } \\
\text { (Barrers) } \\
\mathrm{O}_{2}\end{array}$} \\
\hline & & & $\begin{array}{c}T_{\mathrm{g}} \\
\left({ }^{\circ} \mathrm{C}\right)\end{array}$ & $\begin{array}{c}T_{\gamma} \\
\left({ }^{\circ} \mathrm{C}\right)\end{array}$ & \\
\hline Bis-A & $5 \cdot 15$ & $9 \cdot 91$ & 198 & -84 & $1 \cdot 4$ \\
\hline Bis-MEK & $5: 18$ & 10.01 & 192 & -92 & 1.6 \\
\hline Bis-MIBK & $5 \cdot 21$ & $9 \cdot 56$ & 216 & -72 & $3 \cdot 5$ \\
\hline Bis-Aceto & 5.09 & $9 \cdot 94$ & 267 & -85 & $2 \cdot 1$ \\
\hline Bis-Lev & 5.03 & $10 \cdot 22$ & 165 & -93 & 0.76 \\
\hline
\end{tabular}

All bisphenols polymerized with equimolar mixture of isophthalic and terephthalic acids.

Structure of bisphenols used above as in figure 1

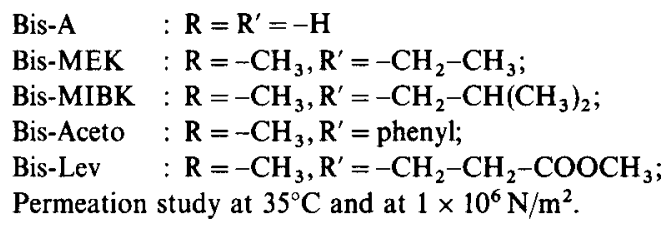

differential scanning calorimetry. Analysis of a systematically varied family of polymers by DMA in particular gives much useful information regarding polymer chain mobility (Charati et al 1994).

Previous studies on the effect of chain rigidity as measured by these transition temperatures $\left(T_{\gamma}\right.$ or $\left.T_{\mathrm{g}}\right)$ have indicated that increasing chain rigidity correlates with reduced permeability (e.g. Light and Seymour 1982). This is generally accepted to be the case for rubbery polymers; however the situation is not so clear for highly glassy polymers. In previous studies the packing density was not kept constant and hence this conclusion may have been premature. Recent work (Houde et al 1995) indicates that in a family of bridge-substituted bisphenol-A-based polyarylates (figure 1), the $d_{\mathrm{sp}}$ is relatively constant and the permeability increases as $T_{y}$, increases (see table 1). More work will be necessary to validate this finding.

\subsection{Simultaneous increase in chain rigidity and decrease in packing density}

Kim et al (1988) have shown the utility of this rule, first developed by Hoehn and Richter (1980), for a series of polyimides. Modifications in polymer structure which lead simultaneously to an increase in chain rigidity and also a decrease in packing density result in an increase in permeability without loss of selectivity. This rule applies equally well to polyarylates and polysulphones (Houde et al 1994).

An example of this rule can be seen by comparing the polyarylates prepared from bisphenol-A and then modified by the tetramethyl substitution (see table 2). The $\mathrm{Me}_{4}$ Bis-A polyarylate is more rigid (higher transition temperatures) and has lower packing density (higher $d_{\mathrm{sp}}$ ). Correspondingly, its permeability is increased threefold without hindering permselectivity. 
Table 2. Physical properties and permeation characteristics of ringsubstituted polyarylates.

\begin{tabular}{lcccc}
\hline Bisphenol & $\begin{array}{c}d \text {-spacing } \\
(\AA)\end{array}$ & $\begin{array}{c}T_{\mathrm{g}} \\
\left({ }^{\circ} \mathrm{C}\right)\end{array}$ & $\begin{array}{c}\text { Permeability } \\
(\text { Barrers }) \\
\mathrm{He}\end{array}$ & $\begin{array}{c}\text { Selectivity } \\
\left(\mathrm{He} / \mathrm{CH}_{4}\right)\end{array}$ \\
\hline Bis-A & $5 \cdot 15$ & 198 & 16 & 40 \\
DMBis-A & $5 \cdot 21$ & 235 & $13 \cdot 6$ & 170 \\
$\mathrm{Me}_{4}$ Bis-A & $5 \cdot 74$ & 274 & 47 & 65 \\
$\mathrm{Br}_{4}$ Bis-A & $4 \cdot 15$ & 285 & $14 \cdot 1$ & 83 \\
\hline
\end{tabular}

All bisphenols polymerized with equimolar mixture of isophthalic and terephthalic acids.

Structure of bisphenols used above as in figure 2

$\begin{array}{ll}\text { Bis-A } & : \mathbf{R}=\mathbf{R}^{\prime}=-\mathrm{H} \\ \mathbf{M e}_{4} \text { Bis-A } & : \mathbf{R}=\mathbf{R}^{\prime}=-\mathrm{CH}_{3} \\ \mathbf{B r}_{4} \text { Bis-A } & : \mathbf{R}=\mathbf{R}^{\prime}=-\mathbf{B r}\end{array}$

Permeation study at $35^{\circ} \mathrm{C}$ and at $1 \times 10^{6} \mathrm{~N} / \mathrm{m}^{2}$.

\subsection{Effect of polarity}

Increased solute-polymer interaction would tend to increase solubility of the solute and thereby increase its permeability (e.g. see Chern et al 1987). Increase in permeability through increasing the polar-group proportion in a polymer would be expected to be particularly effective in the case of gases like $\mathrm{CO}_{2}$ or $\mathrm{O}_{2}$ relative to $\mathrm{CH}_{4}$ or inert gases.

The effect of polarity may be double-edged in that it may also lead to increased polymer interchain interactions causing a decrease in permeability. This can be seen by comparing the tetrabromo and tetramethyl substitutions on the bisphenol- $A$ phenyl rings (table 2).

\section{Insights from molecular modelling}

While molecular modelling cannot be used yet to a priori design polymers for gas separation, it can be used to rationalize and understand the experimental results. As a result of these insights, it can suggest further polymers for synthesis.

The calculations used for obtaining the results described here are done with a conventional software package (CharmM) based on molecular-mechanics approximations. An electron delocalization term was added separately to the energy calculations (Charati et al 1991b).

\subsection{Polyarylate isomers}

Intuitively, it would appear that polyarylates made with terephthalic acid would be more linear and pack denser than the corresponding polyarylates made with isophthalic acid. Actually, as indicated earlier (figure 3), the reverse is true and in general the terephthalic acid-based polymers have higher $d_{\mathrm{sp}}$ and permeability values. 
Modelling shows that the results are reasonable in terms of their three-dimensional structure. While the isophthalic acid and bisphenol-A-based polyarylate has a regular helical structure, the one based on terephthalic acid has a crank-shaft-type configuration. Packing of individual chains into assemblies shows that the isophthalic acid-based polymer chains have a $20 \%$ smaller interchain separation than the terephthalic acid-based polymer (Vetrivel et al 1991). This is the explanation for the data shown in figure 3 .

\subsection{Bridge-substituted polyarylates}

The bridge-carbon-substituted bisphenol-A and isophthalic/terephthalic acid-based polyarylates have relatively constant $d_{\text {sp }}$ values irrespective of the fact that the methyl group is substituted by more bulky groups. Normally, one would expect bulky groups to alter the chain conformation or prevent chains from approaching each other and lead to lower packing density. In this case, in the preferred (energy-minimized) chain conformation, the mono-substituted group is oriented towards the outside of the helix. The chain conformation is not altered significantly and this prevents the $d_{\mathrm{sp}}$ from changing much (see table 1).

\subsection{Phenyl-ring substituted polyarylates}

Energy mapping of the rotational mobility of various dihedral angles in the polymer chain has indicated the importance of the bisphenol phenyl rings in governing the overall chain rigidity (Charati et al 1991b). The rotational mobility of the phenyl rings is important for determining the calculated configurational entropy which correlates well with the observed $T_{\mathrm{g}}$. The phenyl ring mobility has also been related to the $\gamma$ transition in both polyarylates (Charati et al 1994) and polycarbonates (Yee and Smith 1981). The ortho hydrogen repulsion on adjacent phenyl rings is an important variable determining chain conformation. These observations from the molecular modelling work provide a rationalization of the large change in chain rigidity and interchain spacing, and consequently permeability as a result of phenyl ring substitution (table 2).

Results (to be published) indicate that in polyarylates with such substitutions the interchain separation is dominated by the tetra-substituted bisphenol moiety and the effect of the acid group (isophthalic or terephthalic) in determining $d_{\mathrm{sp}}$ is wiped out. The reason for this is the change in chain conformation and decrease in chain colinearity necessitated by accommodating the phenyl ring substituents.

In the case of asymmetrically substituted phenyl rings (dimethylBis-A) the $d_{\mathrm{sp}}$ does not change dramatically; however, the free-volume distribution may be shifted towards lower values. DMBis-A-based polyarylates appear to give higher selectivities than other polyarylates (see table 2). In order to get both high selectivity and high permeability, polymers can be designed with an asymmetrically substituted bisphenol-A to give selectivity and a modified acid which promotes high permeability. A novel polymer based on this principle has recently been synthesized and shown to have an $\mathrm{O}_{2}$ permeability of 1.5 barrers and $\mathrm{O}_{2} / \mathrm{N}_{2}$ selectivity of 8-9 (Jadhav et al 1993). 


\section{Acknowledgements}

The author is grateful to his present and past colleagues at NCL whose joint work is presented here. These include primarily Drs M G Kulkarni, A Y Houde, S C Charati, U K Kharul, J P Jog and S P Vernekar. Financial assistance from the Department of Science and Technology, New Delhi, is also gratefully acknowledged.

\section{References}

Anand J N, Bales S E, Feay D C and Jeanes T O 1989 U. S. Patent 4, 840, 646 Barbari T A, Koros W J and Paul D 1989 J. Membrane Sci. 4269 Charati S G, Houde A Y, Kulkarni S S and Kulkarni M G 1991a J. Polym. Sci., Polym. Phys. Ed. 29921 Charati S G, Vetrivel R, Kulkarni S S and Kulkarni M G 1991b Macromolecules 252215 Charati S G, Jog J P, Kulkarni S S and Kulkarni M G 1994 J. Appl. Polym. Sci. 541093 Chern R T, Sheu F R, Jia L, Stannett V T and Hopfenberg H B 1987 J. Membrane Sci. 35103 Coleman M R and Koros W J $1990 \mathrm{~J}$. Membrane Sci. 50285 Hoehn H and Richter J W 1980 U.S. Patent reissue 30, 351

Houde A Y, Kulkarni S S and Kulkarni M G 1992 J. Membrane Sci. 71117 Houde A Y, Kulkarni S S and Kulkarni M G 1994 J. Membrane Sci. 95147 Houde A Y, Kulkarni S S, Kharal U K, Charati S G and Kulkarni M G 1995 J. Membrane Sci. (in press) Jadhav A S, Purshottam J, Kulkarni S S and Vernekar S P 1993 Indian Patent application 588/DEL/93 Kim T H, Koros W J, Husk G R and O'Brien K C 1988 J. Membrane Sci. 3745 Kulkarni S S and Stern S A 1983 J. Polym. Sci., Polym. Phys. Ed. 21441

Light R R and Seymour R W 1982 Polym. Engg. Sci. 22857

Matsumoto K, Xu P and Nishikimi T 1993 J. Membrane Sci. 8115

McHattie J S, Koros W J and Paul D 1991 Polymer 32840

Muruganandam N, Koros W J and Paul D R 1987 J. Polym. Sci., Polym. Phys. Ed. 251999

Pace R J and Datyner A J 1979 J. Polym. Sci., Polym. Phys. Ed. 171675

Salame M 1967 Am. Chem. Soc. Preprints 8137

Schmidhauser J C and Longley K L 1990 J. Appl. Polym. Sci. 392083

Sheu F R and Chern R T 1989 J. Polym. Sci., Polym. Phys. Ed. 271121

Stern S A, Mi Y, Yamamoto H and St Clair A K 1989 J. Polym. Sci., Polym. Phys. Ed. 271887

Vetrivel R, Charati S, Kulkarni S S and Schultz J 1991 Polymer Science, contemporary themes (ed.) S Sivram (New Delhi: Tata-McGraw Hill) vol. II, p. 630

Yee A F and Smith S A 1981 Macromolecules 1454 